\title{
Transparent nonlinear networks
}

\author{
J.R. Yusupov ${ }^{1}$, K.K. Sabirov ${ }^{2}$, M. Ehrhardt ${ }^{3}$ and D.U. Matrasulov ${ }^{1}$ \\ ${ }^{1}$ Turin Polytechnic University in Tashkent, 17 Niyazov Str., 100095, Tashkent, Uzbekistan \\ ${ }^{2}$ Tashkent University of Information Technologies, \\ 108 Amir Temur Str., 100200, Tashkent Uzbekistan \\ ${ }^{3}$ Bergische Universität Wuppertal, Gaußstrasse 20, D-42119 Wuppertal, Germany
}

\begin{abstract}
We consider the reflectionless transport of solitons in networks. The system is modeled in terms of the nonlinear Schrödinger equation on metric graphs, for which transparent boundary conditions at the branching points are imposed. This allows to derive simple constraints, which link equivalent usual Kirchhoff-type vertex conditions to the transparent ones. Our approach is applied to a metric star graph. An extension to more complicated graph topologies is straight forward.
\end{abstract}

\section{INTRODUCTION}

Modeling of soliton dynamics in branched structures and networks is relevant to many practically important tasks arising in optics, fluid dynamics, condensed matter, biological physics and polymers. The motivation for such task arises from the fact that highly efficient transfer of information, charge, heat, spin and optical signals in the form of solitons requires developing of effective models providing tools for tunable wave transport in given lowdimensional materials. Therefore the problem of soliton transport in branched structures attracted much attention recently [1 18$]$.

An effective model that can be used for modeling of soliton dynamics in networks is based on the solution of nonlinear wave equations on metric graphs. These metric graphs are the set of bonds (each assigned a length) connected to each other according to a rule, which is called topology of a graph. Solving the wave equation in such domain requires imposing boundary conditions both, at the branching points (vertices) and ends of each branch. During the past decade, different nonlinear wave equations on networks have become one of the rapidly developing topics both, in theoretical and mathematical physics. The early study of the nonlinear Schrödinger equation (NLSE) and soliton dynamics in networks dates back to the Refs. [1, 2], where the integrability of NLSE under certain constraint have been shown. Later such study has been extended to NLSE on planar graphs 7], sine-Gordon [10], nonlinear Dirac [14] and nonlinear heat [16] equations. Detailed study of corresponding stationary problems was presented in the Refs. [4, 9, 11, 13].

A very important feature of the wave transport in networks is the transmission of solitons through the network branching points, which is usually accompanied by the reflection (backscattering) of a wave at these points. If reflection dominates compared to transmission "resistivity" of a network with respect to the wave propagation becomes large and this makes such network less effective for the use of signal transfer. Therefore, it is quite important from the viewpoint of practical applications, to reduce such resistivity by providing a minimum of reflection, or by its absence. This task leads to the problem of tunable soliton transport in networks, whose ideal re- sult should be reflectionless transmission of the waves through the branching points of the structure. For the practical applications in condensed matter, such transmission implies ballistic transport of charge, spin, heat and other carriers in low-dimensional branched materials. The latter is of importance for the functionalization of low-dimensional materials having branched structure.

Reflectionless transport of solitons in optical fiber networks is another important problem for the fiber optics, as many information-communication devices (e.g., computers, computer networks, telephones, etc.) use solitons for information (signal) transfer. Such networks are also used in different optoelectronic devices. High speed and lossless transfer of information in such devices require minimum of backscattering or its absence. Important areas, where the reflectionless or ballistic transport of optical solitons in networks is required, are molecular electronics and conducting polymers [17].

Earlier, possibility for reflectionless transmission of solitons in networks has been considered in some studies. In particular, it was found in the Refs. [1, 2] that the transmission of solitons through the network branching point can be reflectionless, provided certain constraints are fulfilled. It was shown also that these constraints provide the integrability of NLS equation on networks. Later, a similar effect was observed for other nonlinear PDEs, such as the sine-Gordon equation Refs. [10] and the nonlinear Dirac equation Refs. [14]. In other words, the above studies revealed a conjecture (at least, for few PDE), which states that if nonlinear wave equation on a network is integrable, then the transmission of solitons through the branching points becomes reflectionless. However, no strict explanation for such conjecture have been presented in those studies.

In this paper we give a proof of the above conjecture by showing that the constraints providing such reflectionless transmission and integrability of the nonlinear Schrödinger equation on networks, link equivalent usual Kirchhoff type vertex boundary conditions to the socalled transparent boundary conditions. These latter conditions are well studied previously in detail in the Refs. 21 36.

The paper is organized as follows. In the next section we briefly recall the concept of transparent boundary con- 
ditions for the nonlinear Schrödinger equation on a line. Section III provides extension of the concept of transparent boundary conditions to solitons in networks described by the nonlinear Schrödinger equation on metric graphs and presents some numerical results. Finally, Section IV presents some concluding remarks.

\section{TRANSPARENT BOUNDARY CONDITIONS FOR THE NONLINEAR SCHRÖDINGER EQUATION ON A LINE}

The problem of transparent boundary conditions (TBC) for the linear partial differential equations (PDE) is well developed topic in mathematical and theoretical physics (see, e.g., 21 36 for review). However, despite such progress, for the nonlinear PDE, the topic is not well established, yet, due to certain complications of the problem in nonlinear case. One of the effective approaches for the case is considering the nonlinear term as potential in linear PDE and called "potential approach". Below we briefly recall this approach following the Refs. [37, 38].

We consider the wave (particle) motion in a 1D domain $(-\infty,+\infty)$ described by the following time-dependent nonlinear Schrödinger equation:

$$
i \partial_{t} \psi+\partial_{x}^{2} \psi+\beta|\psi|^{2} \psi
$$

with the initial condition

$$
\psi(x, 0)=\psi_{0}(x) .
$$

Derivation of transparent boundary conditions for nonlinear case is rather complicated than that for the linear one. However, one can use so-called potential approach, where Eq. (1) can be considered as the linear Schrödinger equation with the potential $V=\beta|\psi|^{2}$. Then, one can rewrite Eq. (1) in a "linear form" as

$$
i \partial_{t} \psi+\partial_{x}^{2} \psi+V \psi=0
$$

Let us denote by $\psi$ the solution of Eq. (3) and by $v$ the new unknown defined by the relation

$$
v(x, t)=e^{-i \nu(x, t)} \psi(x, t),
$$

where

$$
\nu(x, t)=\int_{0}^{t} V(x, s) d s .
$$

For the time- and space-derivative of $\psi$ we have

$$
\begin{gathered}
i \partial_{t} \psi=e^{i \nu}\left(i \partial_{t}-V\right) v \\
\partial_{x}^{2} \psi=i e^{i \nu}\left(\partial_{x}^{2} v+2 i \partial_{x} \nu \cdot \partial_{x} v+i \partial_{x}^{2} \nu \cdot v-\left(\partial_{x} \nu\right)^{2} v\right) .
\end{gathered}
$$

It is clear that the function $v$ satisfies the Schrödinger equation

$$
L\left(x, t, \partial_{x}, \partial_{t}\right) v=i \partial_{t} v+\partial_{x}^{2} v+A \partial_{x} v+B v=0,
$$

where $A=2 i \partial_{x} \nu$ and $B=i \partial_{x}^{2} \nu-\left(\partial_{x} \nu\right)^{2}$.

Expanding the factorization (8), we get

$$
\begin{aligned}
L & =\left(\partial_{x}+i \Lambda^{-}\right)\left(\partial_{x}+i \Lambda^{+}\right) \\
& =\partial_{x}^{2}+i\left(\Lambda^{-}+\Lambda^{+}\right) \partial_{x}+i O p\left(\partial_{x} \lambda^{+}\right)-\Lambda^{-} \Lambda^{+},
\end{aligned}
$$

where $\Lambda^{ \pm}=\Lambda^{ \pm}\left(x, t, \partial_{t}\right)$ are classical pseudodifferential operators, $\lambda_{1 / 2}^{ \pm}$are the principal symbols of operators $\Lambda^{ \pm}$given by $\lambda_{1 / 2}^{ \pm}=\mp \sqrt{-\tau}$ and the function $\tau$ is inhomogeneous of degree 1 and is an element of $S_{S}^{1 / 2}$. The total symbol $\lambda^{ \pm}=\sigma\left(\Lambda^{ \pm}\right)$of $\Lambda^{ \pm}$admits an asymptotic expansion in inhomogeneous symbols as

$$
\lambda^{ \pm}=\sigma\left(\Lambda^{ \pm}\right) \sim \sum_{j=0}^{+\infty} \lambda_{1 / 2-j / 2}^{ \pm}
$$

From (9) we deduce the system of operators

$$
\begin{gathered}
i\left(\Lambda^{-}+\Lambda^{+}\right)=A \\
i O p\left(\partial_{x} \lambda^{+}\right)-\Lambda^{-} \Lambda^{+}=i \partial_{t}+B,
\end{gathered}
$$

which yields the following symbolic system of equations:

$$
i\left(\lambda^{+}+\lambda^{-}\right)=A
$$

$$
i \partial_{x} \lambda^{+}-\sum_{\alpha=0}^{+\infty} \frac{(-i)^{\alpha}}{\alpha !} \partial_{\tau}^{\alpha} \lambda^{-} \partial_{t}^{\alpha} \lambda^{+}=-\tau+B
$$

If we identify the terms of order $1 / 2$ in the Eq. (13), we obtain $\lambda_{1 / 2}^{-}=-\lambda_{1 / 2}^{+}$. Then from Eq. (14), we get

$$
\lambda_{1 / 2}^{+}= \pm \sqrt{-\tau}
$$

The Dirichlet-Neumann operator corresponds to the choice $\lambda_{1 / 2}^{+}=-\sqrt{-\tau}$. From the factorization (8) we have the following transparent boundary condition applied to the unknown wave function $v$

$$
\begin{aligned}
\left(-\partial_{x}+i \Lambda^{+}\right) v(0, t) & =0 \\
\left(\partial_{x}+i \Lambda^{+}\right) v(L, t) & =0 .
\end{aligned}
$$

Then using Eq. (4) the formal transparent boundary conditions for $\psi$ at $x=0$ and $x=L$ can be written as 37.

$$
\begin{gathered}
-\partial_{x} \psi(0, t)+e^{-i \frac{\pi}{4}} e^{i \nu(0, t)} \partial_{t}^{1 / 2}\left(e^{-i \nu(0, t)} \psi(0, t)\right)=0 \\
\partial_{x} \psi(L, t)+e^{-i \frac{\pi}{4}} e^{i \nu(L, t)} \partial_{t}^{1 / 2}\left(e^{-i \nu(L, t)} \psi(L, t)\right)=0
\end{gathered}
$$

where

$$
\partial_{t}^{1 / 2} f(t)=\frac{1}{\sqrt{\pi}} \partial_{t} \int_{0}^{t} \frac{f(s)}{\sqrt{t-s}} d s
$$


Formally, Eqs. (18) and (19) are similar to those for the linear case. We remark that a detailed treatment of Eqs. (11), (2), (18) and (19) can be found in the Refs. [37, 38], where the discretization scheme and the numerical method for solving of this problem are also presented. We note that the boundary conditions (18) and (19) are true both, for focusing $(\beta>0)$ and defocusing $(\beta<0)$ cases. In the next Section III we will modify these boundary conditions for the nonlinear Schrödinger equation on metric graphs.

\section{TRANSPARENT BOUNDARY CONDITIONS FOR NLSE ON METRIC GRAPHS}

Soliton dynamics in networks is one of the rapidly evolving topics during past decade. The early treatment of the problems dates back to the Ref. [1], where soliton solutions of the nonlinear Schrödinger equation on metric graphs was obtained and integrability of the problem under certain constraints was shown by proving the existence of infinite number of conserving quantities. An interesting feature found in [1] was the fact that for integrable case, transmission of solitons through the graph vertices is reflectionless, i.e. there is no backscattering of solitons at the graph branching point. An explanation of such effect was given in recent papers [12, 19], where it was strictly shown that if the parameters of the generalized Kirchhoff boundary conditions on a star graph are related to the parameters of the nonlinear evolution equations and satisfy a single constraint, then the nonlinear evolution equation on the star graph can be reduced to the homogeneous equation on the infinite line. Here we provide more strict proof of this conjecture, by showing that vertex boundary conditions in the form of weight continuity and generalized Kirchhoff rules become equivalent to transparent boundary conditions, if the parameters of the problem fulfill the integrability condition given in the form of the sum rule. To do this, we will apply the above method for imposing transparent boundary conditions to the NLSE on metric graphs. Before doing this, let us briefly recall the treatment of the NLS equation on metric graphs following Ref. [1].

Before this was done for quantum graphs described by the linear Schrödinger equation on metric graphs. We consider the star graph with three bonds $B_{j}$ (see, Fig. 1) for which a coordinate $x_{j}$ is assigned. Choosing the origin of coordinates at the vertex, 0 for bond $B_{1}$ we put $x_{1} \in$ $(-\infty, 0]$ and for $B_{1,2}$ we fix $x_{2,3} \in[0,+\infty)$. In what follows we use the notation $\Psi_{j}(x)$ for $\Psi_{j}\left(x_{j}\right)$ and $x$ is the coordinate on the bond $j$ to which the component $\Psi_{j}$ refers. The nonlinear Schrödinger equation on each bond of such graph can be written as

$$
i \partial \psi_{j}+\partial_{x}^{2} \psi_{j}+\beta_{j}\left|\psi_{j}\right|^{2} \psi_{j}=0
$$

Solving this equation (21) requires imposing initial conditions and boundary conditions at the branching

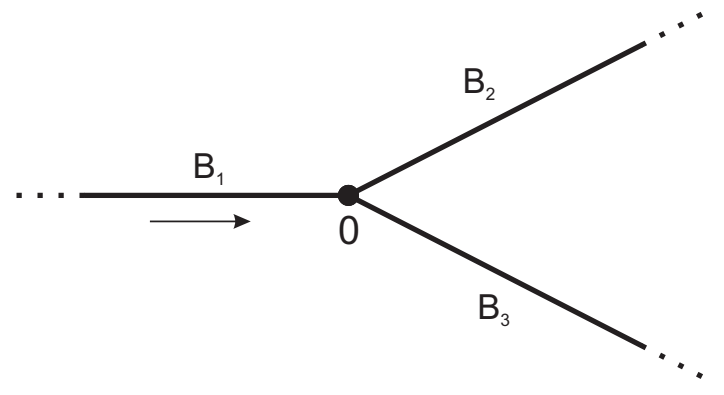

FIG. 1: Sketch of a star graph with 3 semi-infinite bonds.

point. The latter can be derived from the fundamental physical laws, such as energy and norm conservation, which are given as

$$
\frac{d N}{d t}=\frac{d E}{d t}=0
$$

where

$$
N(t)=\int_{-\infty}^{0}\left|\psi_{1}\right|^{2} d x+\int_{0}^{\infty}\left|\psi_{2}\right|^{2} d x+\int_{0}^{\infty}\left|\psi_{3}\right|^{2} d x
$$

and

$$
E=E_{1}+E_{2}+E_{3}
$$

with

$$
E_{k}=\int_{B_{k}}\left[\left|\frac{\partial \psi_{k}}{\partial x}\right|^{2}-\frac{\beta_{k}}{2}\left|\psi_{k}\right|^{4}\right] d x
$$

The conservation laws Eq. (22) lead to the following vertex conditions [1]

$$
\alpha_{1} \psi_{1}(0)=\alpha_{2} \psi_{2}(0)=\alpha_{3} \psi_{3}(0)
$$

and generalized Kirchhoff rules

$$
\left.\frac{1}{\alpha_{1}} \frac{\partial \psi_{1}}{\partial x}\right|_{x=0}=\left.\frac{1}{\alpha_{2}} \frac{\partial \psi_{2}}{\partial x}\right|_{x=0}+\left.\frac{1}{\alpha_{3}} \frac{\partial \psi_{3}}{\partial x}\right|_{x=0}
$$

where $\alpha_{j}$ are nonzero real constants. The asymptotic conditions for Eq. (21) are imposed as

$$
\left.\psi_{j}\right|_{|x| \rightarrow+\infty} \rightarrow 0 .
$$

The single soliton solutions of Eq. (21) fulfilling the vertex boundary conditions (23), (24) and the asymptotic condition, (25) can be written as [1]

$$
\psi_{j}(x, t)=a \sqrt{\frac{2}{\beta_{j}}} \frac{\exp \left[i \frac{v x}{2}-i\left(\frac{v^{2}}{4}-a^{2}\right) t\right]}{\cosh [a(x-l-v t)]},
$$

where the parameters $\beta_{j}$ fulfill the sum rule

$$
\frac{1}{\beta_{1}}=\frac{1}{\beta_{2}}+\frac{1}{\beta_{3}} \text {. }
$$



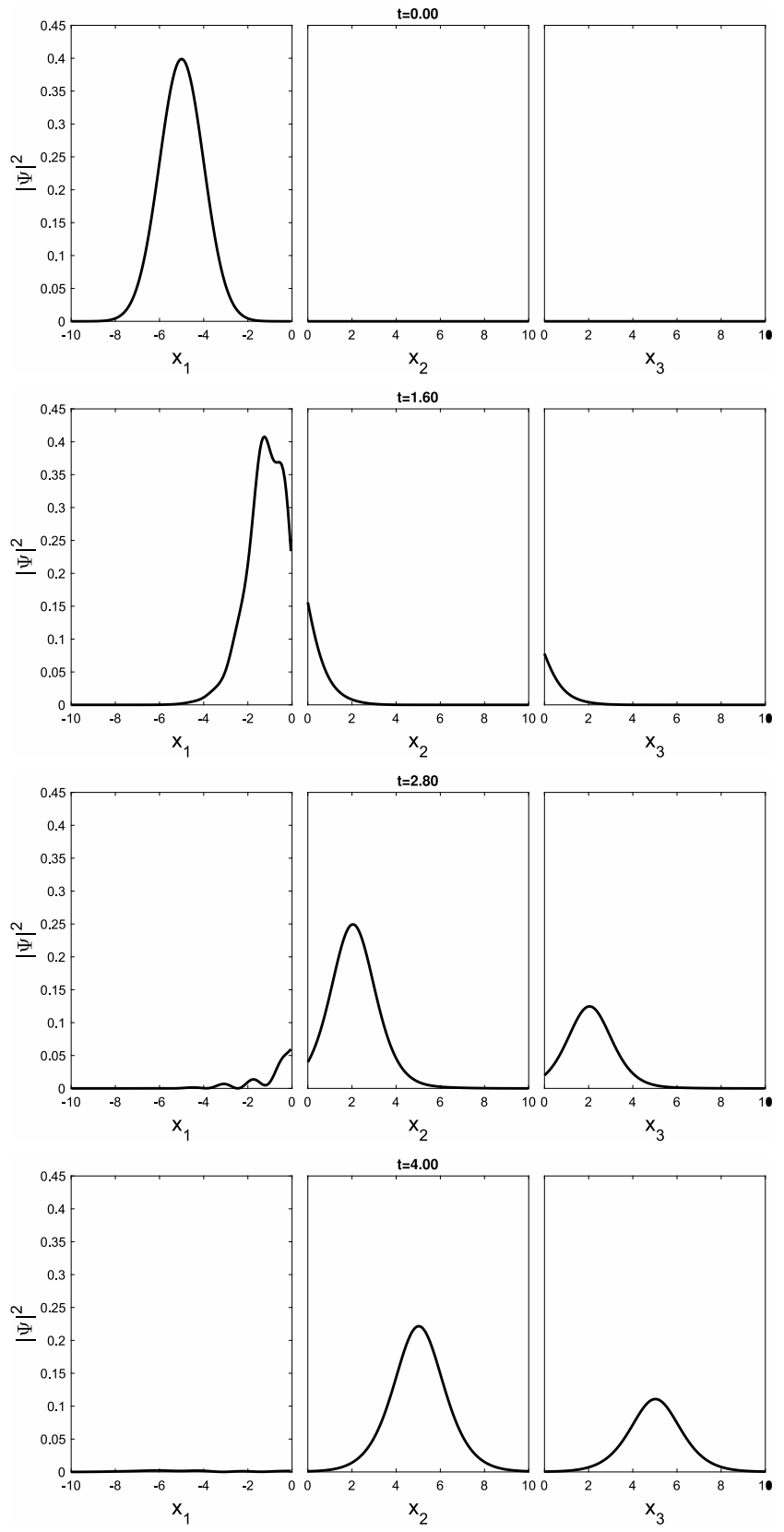

FIG. 2: The profile of the wave function plotted at different time moments for the regime when the sum rule is fulfilled (no reflection is occurred): $\alpha_{1}=\sqrt{\beta_{1}}=1 / \sqrt{1 / 2+1 / 4}, \alpha_{2}=$ $\sqrt{\beta_{2}}=\sqrt{2}$ and $\alpha_{3}=\sqrt{\beta_{3}}=\sqrt{4}$. Each column number (from the left to the right) corresponds to a bond number.

Here $v, l$ and $a$ are bond-independent parameters characterizing velocity, initial center of mass and amplitude of a soliton, respectively.

Eq. (27) presents the conditions for integrability of the problem given by Eqs. (21), (23), (24) and (25), i.e. the integrability of the nonlinear Schrödinger equation on a metric star graph presented in Fig. 1. It was shown in [1] that under the constraint (27) the problem has an infinite number of constants of motion. Below we show an additional consequence following from Eq. (27), which can be formulated as follows: If the parameters $\beta_{j}$ in Eq. (21) fulfill the condition (constraint) (27), then the vertex boundary conditions (23) and (24) become equivalent to transparent boundary conditions at the point 0 .

Without loss of generality of the approach, we can assume that $\alpha_{j}=\sqrt{\beta_{j}}$. To impose transparent boundary conditions for the NLS equation on metric graph shown in Fig. 1, we split the whole domain (graph) into two domains called "interior" $(-\infty<x<0)$ and "exterior" $(0<x<\infty)$ ones (see, e.g., Refs. 20, 22 25] for details). Correspondingly, we have interior and exterior problems. The interior problem is given on $B_{1}$ by the equations

$$
\begin{aligned}
i \partial \psi_{1}+\partial_{x}^{2} \psi_{1}+\beta_{1}\left|\psi_{1}\right|^{2} \psi_{1} & =0, \quad x<0, t>0 \\
\left.\psi_{1}\right|_{t=0} & =\Psi^{I}(x), \\
\left.\partial_{x} \psi_{1}\right|_{x=0} & =\left.\left(T_{+} \psi_{1}\right)\right|_{x=0} .
\end{aligned}
$$

The exterior problems for $B_{2,3}$ can be written as

$$
\begin{aligned}
i \partial \psi_{2,3}+\partial_{x}^{2} \psi_{2,3}+\beta_{2,3}\left|\psi_{2,3}\right|^{2} \psi_{2,3} & =0 \\
\left.\psi_{2,3}\right|_{t=0} & =0 \\
\left.\psi_{2,3}\right|_{x=0}=\Phi_{2,3}(t), \Phi_{2,3}(0) & =0 \\
\left.\left(T_{+} \Phi_{2,3}\right)\right|_{x=0} & =\left.\partial_{x} \psi_{2,3}\right|_{x=0}
\end{aligned}
$$

We rewrite the NLSE of exterior problems for $B_{2,3}$ as

$$
i \partial \psi_{2,3}+\partial_{x}^{2} \psi_{2,3}+V_{2,3} \psi_{2,3}=0
$$

with the potentials $V_{2,3}=\beta_{2,3}\left|\psi_{2,3}\right|^{2}$. Furthermore, we introduce the new functions $v_{2,3}$ given as

$$
v_{2,3}(x, t)=e^{-i \nu_{2,3}(x, t)} \psi_{2,3}(x, t)
$$

where

$$
\nu_{2,3}(x, t)=\int_{0}^{t} V_{2,3}(x, s) d s .
$$

Then from the factorization in Eq. (9) we have the following transparent boundary conditions for the wave functions $v_{2,3}$ :

$$
\left(-\partial_{x}+i \Lambda^{+}\right) v_{2,3}(0, t)=0 .
$$

Using Eq. (29) we can write the formal transparent boundary conditions for $\psi_{2,3}$ at $x=0$ as

$$
\begin{aligned}
-\partial_{x} \psi_{2,3}(0, t)+ & e^{-i \frac{\pi}{4}} e^{i \nu_{2,3}(0, t)} \\
& \partial_{t}^{1 / 2}\left(e^{-i \nu_{2,3}(0, t)} \psi_{2,3}(0, t)\right)=0 .
\end{aligned}
$$


Using the vertex boundary condition (23) we have

$$
\begin{aligned}
\left.\partial_{x} \psi_{2,3}\right|_{x=0}= & \frac{1}{\sqrt{\pi}} e^{-i \frac{\pi}{4}+i \beta_{2,3} \int_{0}^{t}\left|\psi_{2,3}(0, s)\right|^{2} d s} . \\
& \partial_{t} \int_{0}^{t} \frac{\psi_{2,3}(0, \tau) e^{-i \beta_{2,3} \int_{0}^{\tau}\left|\psi_{2,3}(0, s)\right|^{2} d s}}{\sqrt{t-\tau}} d \tau \\
= & \frac{1}{\sqrt{\pi}} \sqrt{\frac{\beta_{1}}{\beta_{2,3}} e^{-i \frac{\pi}{4}+i \beta_{1} \int_{0}^{t}\left|\psi_{1}(0, s)\right|^{2} d s}} \\
& \partial_{t} \int_{0}^{t} \frac{\psi_{1}(0, \tau) e^{-i \beta_{1} \int_{0}^{\tau}\left|\psi_{1}(0, s)\right|^{2} d s}}{\sqrt{t-\tau}} d \tau .
\end{aligned}
$$

From the vertex boundary condition (24) and (33) we get

$$
\begin{aligned}
\left.\partial_{x} \psi_{1}\right|_{x=0}= & \left.\frac{\sqrt{\beta_{1}}}{\sqrt{\beta_{2}}} \partial_{x} \psi_{2}\right|_{x=0}+\left.\frac{\sqrt{\beta_{1}}}{\sqrt{\beta_{3}}} \partial_{x} \psi_{3}\right|_{x=0} \\
= & \frac{1}{\sqrt{\pi}} \beta_{1}\left(\frac{1}{\beta_{2}}+\frac{1}{\beta_{3}}\right) e^{-i \frac{\pi}{4}+i \beta_{1} \int_{0}^{t}\left|\psi_{1}(0, s)\right|^{2} d s} . \\
& \partial_{t} \int_{0}^{t} \frac{\psi_{1}(0, \tau) e^{-i \beta_{1} \int_{0}^{\tau}\left|\psi_{1}(0, s)\right|^{2} d s}}{\sqrt{t-\tau}} d \tau .
\end{aligned}
$$

It is clear that if the sum rule given by Eq. (27) is fulfilled, i.e.,

$$
\beta_{1}\left(\frac{1}{\beta_{2}}+\frac{1}{\beta_{3}}\right)=1
$$

then the boundary condition given by Eq. (34) coincides with that in Eq. (19). Thus fulfilling the sum rule (27) implies that vertex boundary conditions (23) and (24) become equivalent to transparent boundary conditions at the graph vertex. This can be shown by direct numerical solution of Eq. (21) for the boundary conditions (23) and (24). In Fig. 2 the profile of the soliton $\left|\psi_{j}\right|^{2}$ obtained numerically is plotted at different time moments for the regime, when the sum rule (27) is fulfilled. Numerical simulations are performed for the right traveling Gaussian wave packet given by

$$
\Psi^{I}(x)=(2 \pi)^{-1 / 4} \exp \left(2.5 i x-(x+5)^{2} / 4\right)
$$

at four consecutive time steps.

To show that for the case, when the sum rule is broken the transmission of soliton is accompanied by reflections, we plotted the reflection coefficient, which is determined as the ratio of the partial norm for the first bond to the total norm

$$
R=\frac{N_{1}}{N_{1}+N_{2}+N_{3}},
$$

as a function of $\alpha_{1}$ for the fixed values of $\alpha_{2}$ and $\alpha_{3}$. It is clear from this plot that the reflection coefficient

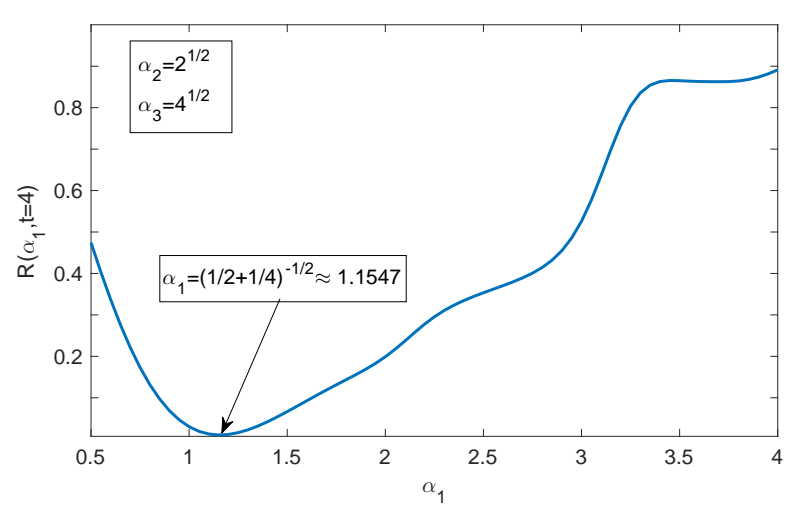

FIG. 3: Dependence of the vertex reflection coefficient $R$ on the parameter $\alpha_{1}$ when time elapses $(t=4)$.

becomes zero at the value of $\alpha_{1}$, which provides fulfilling of the sum rule (27). This also can be considered as additional confirmation for becoming equivalent the vertex boundary conditions in Eqs. (23) and (24) to the transparent ones. It is clear that such conjecture can be derived for star graph with arbitrary number of bonds. Finally, we note that the above constraint for transparent boundary conditions given by Eq. (27) is applicable not only for solitons, but for arbitrary solutions of the NLS equation on graphs.

\section{CONCLUSIONS}

In this paper we studied the problem of reflectionless soliton transport in network branching points by modeling the soliton dynamics in networks in terms of the nonlinear Schrödinger equation on metric graphs. By combining the concept of transparent boundary conditions with the Kirchhoff-type boundary conditions at the vertex, we derived constraints, which make equivalent the latter to transparent ones. This gives clear explanation for the previously observed (see, [1]) conjecture for the absence of soliton backscattering, when the NLS equation on metric graphs is integrable and integrability if provided in terms of the above constraint.

Also, solving the problem numerically, we have shown for the star graph a reflectionless transmission of soliton through the vertex in the case of fulfilling of the sum rule by the parameters. We note that the approach can be directly extended to arbitrary graph topologies, which contain any subgraph connected to two or more outgoing, semi-infinite bonds. Moreover, we believe that approach can be extended to other PDE, where similar regime of reflectionless vertex transmission of sine-Gordon [10] and Dirac 14] solitons have been observed. We note that the approach used in this paper can be directly extended to other graphs topologies, such as tree, loop, triangle, etc., provided the graph consists of finite subgraph and two or more semiinfinite outgoing bonds. 
The above model for reflectionless soliton transport through the network branching points may have direct and important applications for different practically important problems of optics, condensed matter and polymers. Among such applications one can consider optical fiber networks widely used in computing and communication technologies, where the signal transfer is done in the form of soliton transport.

\section{ACKNOWLEDGEMENTS}

This work is partially supported by the grant of the Ministry for Innovation Development of Uzbekistan (Ref. Nr. BF2-022).
[1] Z. Sobirov, D. Matrasulov, K. Sabirov, S. Sawada, and K. Nakamura, Phys. Rev. E 81, 066602 (2010).

[2] Z. Sobirov, D. Matrasulov, S. Sawada, and K. Nakamura, Phys. Rev. E 84, 026609 (2011).

[3] R. Adami, C. Cacciapuoti, D. Finco, D. Noja, Rev. Math. Phys, 234 (2011).

[4] K.K. Sabirov, Z.A. Sobirov, D. Babajanov, and D.U. Matrasulov, Phys. Lett. A, 377, 860 (2013).

[5] J.-G. Caputo, D. Dutykh, Phys. Rev. E 90, 022912 (2014).

[6] D. Noja, Philos. Trans. R. Soc. A 372, 20130002 (2014).

[7] H. Uecker, D. Grieser, Z. Sobirov, D. Babajanov and D. Matrasulov, Phys. Rev. E 91, 023209 (2015).

[8] D. Noja, D. Pelinovsky, and G. Shaikhova, Nonlinearity 28, 2343 (2015).

[9] R. Adami, C. Cacciapuoti, D. Noja, J. Diff. Eq. 260, 7397 (2016).

[10] Z. Sobirov, D. Babajanov, D. Matrasulov, K. Nakamura, and H. Uecker, EPL 115, 50002 (2016).

[11] R. Adami, E. Serra, P. Tilli, Commun. Math. Phys. 352, 387 (2017).

[12] A. Kairzhan, D.E. Pelinovsky, J. Phys. A: Math. Theor. 51, 095203 (2018).

[13] K.K. Sabirov, S. Rakhmanov, D. Matrasulov and H. Susanto, Phys. Lett. A, 382, 1092 (2018).

[14] K.K. Sabirov, D.B. Babajanov, D.U. Matrasulov and P.G. Kevrekidis, J. Phys. A: Math. Gen. 51435203 (2018).

[15] K.K. Sabirov, M. Akromov, Sh.R. Otajonov, D.U. Matrasulov, arXiv:1808.10751.

[16] K. Sabirov, Zh. Zhunussova, D. Babajanov, D. Matrasulov, arXiv:1806.10957.

[17] D. Babajanov, H. Matyoqubov, D. Matrasulov, J. Chem. Phys., 149, 164908 (2018).

[18] D. Noja, S. Rolando, S. Secchi, J. Diff. Eqn. 266, 147 (2019).

[19] A. Kairzhan, D. E. Pelinovsky, R. H. Goodman, arXiv:1902.03612.
[20] J. R. Yusupov, K. K. Sabirov, M. Ehrhardt and D. U. Matrasulov, arXiv:1812.03736

[21] A. Arnold and M. Ehrhardt, J. Comput. Phys., 145(2), 611-638 (1998).

[22] M. Ehrhardt, VLSI Design, 9(4), 325-338 (1999).

[23] M. Ehrhardt and A. Arnold, Riv. di Math. Univ. di Parma, 6(4), 57-108 (2001).

[24] M. Ehrhardt, Acta Acustica united with Acustica, 88, 711-713 (2002).

[25] A. Arnold, M. Ehrhardt and I. Sofronov, Comm. Math. Sci., 1(3), 501-556 (2003).

[26] S. Jiang, L. Greengard, Comput. Math. Appl., 47, 955 (2004).

[27] X. Antoine, A. Arnold, C. Besse, M. Ehrhardt and A. Schädle, Commun. Comput. Phys. 4(4), 729-796 (2008).

[28] M. Ehrhardt, Appl. Numer. Math. 58(5), 660-673 (2008).

[29] L. Sumichrast and M. Ehrhardt, J. Electr. Engineering 60(2), 301-312 (2009).

[30] X. Antoine et al., J. Comput. Phys., 228(2), 312-335 (2009).

[31] M. Ehrhardt, Numer. Math.: Theo. Meth. Appl. 3(3), 295-337 (2010).

[32] P. Klein, X. Antoine, C. Besse and M. Ehrhardt, Commun. Comput. Phys. 10(5), 1280-1304 (2011).

[33] A. Arnold, M. Ehrhardt, M. Schulte and I. Sofronov, Commun. Math. Sci. 10(3), 889-916 (2012).

[34] R.M. Feshchenko and A.V. Popov, Phys. Rev. E 88, 053308 (2013).

[35] X. Antoine et al., J. Comput. Phys., 277, 268-304 (2014).

[36] P. Petrov and M. Ehrhardt, J. Comput. Phys. 313, 144158 (2016).

[37] X. Antoine, Ch. Besse, S. Descombes, SIAM J. Numer. Anal., 43, 2272 (2006).

[38] A. Zisowsky, M. Ehrhardt, Math. and Comput. Modell., 47, 1264 (2008). 